doi: 10.2306/scienceasia1513-1874.2013.39S.042

\title{
Numerical simulations of an SIR epidemic model with random initial states
}

\author{
Almbrok Hussin Alsonosi Omar*, Yahya Abu Hasan \\ School of Mathematical Sciences, Universiti Sains Malaysia, 11800 Penang, Malaysia \\ *Corresponding author, e-mail: almabrok76@yahoo.com
}

Received 7 Jan 2013

Accepted 5 Apr 2013

\begin{abstract}
When the initial state of an epidemic is uncertain, mathematical descriptions of that epidemic in terms of initial conditions must be modified by regarding these initial conditions as random variables having particular distribution functions. In this paper we assume a beta distribution as the initial proportion of infected in an SIR epidemic model. Numerical simulations are carried out on the various classes of the model as the uncertainties are propagated. The probability density functions of the random solutions of these classes over time are also calculated numerically. Some properties of the random solution and the effect of the parameters of the beta distribution on the behaviour of the epidemic are investigated.
\end{abstract}

KEYWORDS: initial value problem, uncertainty, beta distribution

\section{INTRODUCTION}

Many mathematical modelling scenarios involve an inherent level of uncertainty. Naturally there are differences between a mathematical model and reality due to inherent uncertainties in the model. This can happen due to insufficient knowledge about particular components of the model and the proper values for the parameters that are a part of the mathematical model as well as the assumptions adopted during the modelling process are not completely true. A situation can also arise when the initial states are not clearly known. In the SIR epidemic model, uncertainty can happen when the transmission rates are not known with certain or are approximated or when the simulation of an epidemic might require an educated guess for the initial state of infected individuals. In this situation, the mathematical model is not able to describe the dynamic behaviour of the epidemic completely. Hence, mathematical descriptions of the model must be modified to account for uncertainty. Randomness is a basic type of objective uncertainty and in system theory uncertainty is classically treated in probabilistic form by the theory of stochastic processes. There are many ways to incorporate randomness into quantitative modelling and simulation. Kegan and West ${ }^{1}$ investigated the effect of random initial conditions on the simple deterministic model for the susceptible-infectious epidemic. They assumed a beta distribution on the initial proportion of susceptible. Pollett et $\mathrm{al}^{2}$. also addressed this problem and they presented a general method for incorporating random initial conditions in population models where a deterministic model is sufficient to describe the dynamics of the population. Enszer and Stadtherr ${ }^{3}$ assumed intervals as the uncertainties in parameters and initial conditions of SIRS model and other variations of the Kermack-McKendrick models. They used a method to determine mathematical and computational guaranteed bounds on the population trajectories that are possible for given bounds on the uncertain quantities.

In this paper, we assume a beta distribution as the random initial state of the infected class in the SIR model and investigate the effect of random initial state on the dynamic behaviour of the epidemic. This model has no analytical solution, for that, numerical simulations are carried out and some properties of the random solution are discussed.

\section{THE SIR EPIDEMIC MODEL}

The transmission dynamics of infectious disease in a population are considered, among others, in Refs. 4, 5. Most models start off with the basic epidemic model of Kermack and McKendrick ${ }^{6}$. In the model, the population is divided into disjoint classes (compartments). An individual in a population is susceptible when they are healthy, but can become infected (denoted by $S$ ). He is infectious when he is capable of transmitting the infection (denoted by $I$ ). He is in the removed class when he has recovered from the infection or at least temporarily with permanent infectious-acquired immunity or has died because of disease (denoted by $R$ ). Consequently, the SIR epidemic model can be 
written as the following initial value problem:

$$
\begin{array}{rlrl}
\frac{\mathrm{d} S}{\mathrm{~d} t} & =-\frac{\delta I S}{N}, & & S(0)=S_{0} \geqslant 0, \\
\frac{\mathrm{d} I}{\mathrm{~d} t}=\frac{\delta I S}{N}-\gamma I, & I(0) & =I_{0} \geqslant 0, \\
\frac{\mathrm{d} R}{\mathrm{~d} t}=\gamma I, & R(0) & =R_{0}=0,
\end{array}
$$

where $S(t), I(t)$ and $R(t)$ are the numbers in these classes and $N$ is the total population size which is assumed to be fixed (i.e., $S(t)+I(t)+R(t)=N$ for all $t$ ). The parameters $\delta$ and $\gamma$ are the rate of unidirectional transition in the epidemic from susceptible class to infected class and the rate of unidirectional transition in the epidemic from infected class to recovered class, respectively. Dividing (1a), (1b) and (1c) by the constant total population size $N$ yields

$$
\begin{array}{ll}
\frac{\mathrm{d} s}{\mathrm{~d} t}=-\delta i s, & s(0)=s_{0} \geqslant 0, \\
\frac{\mathrm{d} i}{\mathrm{~d} t}=\delta i s-\gamma i, & i(0)=i_{0} \geqslant 0, \\
\frac{\mathrm{d} r}{\mathrm{~d} t}=\gamma i, & r(0)=r_{0}=0,
\end{array}
$$

where $s(t), i(t)$ and $r(t)$ are the fractions in the classes, and $s(t)+i(t)+r(t)=1$.

The SIR epidemic model has been used in different forms for studying epidemiological processes such as the spread of $\mathrm{HIV}^{7}$, influenza ${ }^{8}$ and even smallpox ${ }^{9}$. Indeed, there are various forms of the SIR model including SEIR, SIS, SIRS, and SVIR ${ }^{4}$. Small and $\mathrm{Tse}^{10}$ applied the SPIR model (P for prone) to the spread of SARS.

\section{BETA DISTRIBUTION}

The standard beta distribution gives the probability density of a value $x$ on the interval $(0,1)$ :

$$
\operatorname{Beta}(\alpha, \beta): \operatorname{prob}(x \mid \alpha, \beta)=\frac{x^{\alpha-1}(1-x)^{\beta-1}}{B(\alpha, \beta)}
$$

where $B$ is the beta function

$$
B(\alpha, \beta)=\int_{0}^{1} t^{\alpha-1}(1-t)^{\beta-1} \mathrm{~d} t .
$$

$\alpha$ and $\beta$ are parameters which work together to determine if the distribution has a mode in the interior of the unit interval and whether it is symmetrical. It is clear that when $x \sim \operatorname{Beta}[\alpha, \beta]$ then $(1-$ $x) \sim \operatorname{Beta}[\beta, \alpha]$. This probability density function is a very versatile way to represent outcomes like proportions or probabilities. Hence it is very useful to model uncertainty. In this paper we will consider the initial state of the infected class $\left(i_{0}\right)$ of $(2 \mathrm{~b})$ in the system (2) to be uncertain, where the uncertainty takes the form of a distribution. Since $i$ is a fraction of the total population, the interval $[0,1]$ is its support. Consequently, we will choose the beta distribution as the initial state of the infected class.

In SIR model, the proportion of the total population is fixed at any time and equal to one, and the initial state of the recovered class is equal to zero. Hence the initial state of the susceptible class $\left(s_{0}=1-i_{0}\right)$ is also random and distributed as $\operatorname{Beta}[\beta, \alpha]$. By these assumptions the system (2) becomes

$$
\begin{array}{lrl}
\frac{\mathrm{d} s}{\mathrm{~d} t}=-\delta i s, & s(0) & \sim \operatorname{Beta}[\beta, \alpha], \\
\frac{\mathrm{d} i}{\mathrm{~d} t}=\delta i s-\gamma i, & i(0) \sim \operatorname{Beta}[\alpha, \beta], \\
\frac{\mathrm{d} r}{\mathrm{~d} t}=\gamma i, & r(0)=0 .
\end{array}
$$

\section{THE EFFECT OF RANDOMNESS ON THE SIZE OF AN EPIDEMIC}

Let $\left\{i_{0}^{k} ; k=1, \ldots, m\right\}$ be a random sample distributed as a $\operatorname{Beta}[\alpha, \beta]$. Suppose that $i_{0}^{k}$ is the initial state of (5b) of the infected class. Then $\left\{s_{0}^{k} \mid s_{0}^{k}=\right.$ $\left.1-i_{0}^{k} ; k=1, \ldots, m\right\}$ is the initial state of (5a) of the susceptible class (since $r_{0}^{k}=0$ ). The set $\left\{s_{0}^{k} ; k=\right.$ $1, \ldots, m\}$ is also a random sample distributed as a $\operatorname{Beta}[\beta, \alpha]$.

As we mentioned, (5) cannot be solved analytically. Hence we investigate the system by finding the trajectory of the solution with $t$ being the parameter. Dividing ( $5 \mathrm{~b}$ ) by (5a) we get

$$
\frac{\mathrm{d} i}{\mathrm{~d} s}=\frac{\gamma}{\delta s}-1 .
$$

This equation may be integrated to obtain

$$
i+s-\frac{\gamma}{\delta} \ln s=c
$$

where $c$ is a constant and $\gamma / \delta$ is the basic reproduction ratio denoted by $R_{0}$. It is used to determine whether there will be an epidemic or not. Consider the time run from zero (the start of the epidemic) to $+\infty$ (after the epidemic), and assume a random sample of small numbers $i_{0}^{k} ; k=1, \ldots, m$ becomes infected at the start of the epidemic. Therefore $s_{0}^{k}=1-i_{0}^{k}$ and $r_{0}^{k}=$ 0 for every $k=1, \ldots, m$. Under these considerations, (7) becomes

$$
i^{k}+s^{k}-\frac{\gamma}{\delta} \ln s^{k}=i_{0}^{k}+s_{0}^{k}-\frac{\gamma}{\delta} \ln s_{0}^{k} .
$$


Since $i_{0}^{k}+s_{0}^{k}=1$, (8) becomes

$$
i^{k}=1-s^{k}+\frac{\gamma}{\delta} \ln \frac{s^{k}}{s_{0}^{k}} .
$$

Eq. (9) describes the random trajectories of the model, which shows that $s^{k}$ is always decreasing for all $k=$ $1, \ldots, m$. For the random sample $i^{k}, s^{k}$, we can rewrite (6) as:

$$
\frac{\mathrm{d} i^{k}}{\mathrm{~d} s^{k}}=\frac{\gamma}{\delta s^{k}}-1
$$

Let $\mathrm{d} i^{k} / \mathrm{d} s^{k}=0$. We get $s^{k}=\delta / \gamma$ which is a turning point of the random function in (9). From (10), we can see that, for every $k=1, \ldots, m, i^{k}$ is decreasing for $s^{k}<\delta / \gamma$ and increasing for $s^{k}>\delta / \gamma$. The curves of the function in (10) can be sketched by (9). Now, let the time in both sides of (8) approach infinity. We get

$\lim _{t \rightarrow \infty} i^{k}+\lim _{t \rightarrow \infty} s^{k}-\frac{\gamma}{\delta} \lim _{t \rightarrow \infty} \ln s^{k}=i_{0}^{k}+s_{0}^{k}-\frac{\gamma}{\delta} \ln s_{0}^{k}$.

Let us consider $\lim _{t \rightarrow \infty} i^{k}(t)=0$, and $\lim _{t \rightarrow \infty} s^{k}(t)=s_{\infty}^{k}>0$ as well as $i_{0}^{k}+s_{0}^{k}=1$. Then (11) becomes

$$
s_{\infty}^{k}-\frac{\gamma}{\delta} \ln s_{\infty}^{k}=1-\frac{\gamma}{\delta} \ln s_{0}^{k} .
$$

Eq. (12) describes the proportion of the population that remains susceptible and calculates the proportion of the final size of the epidemic (the proportion of the random sample of the population that has been infected) which is equal to $s_{0}^{k}-s_{\infty}^{k}$.

When $m=1$ the initial condition of the infected class $\left(i_{0}^{k}\right)$ will be a specific number as will the initial condition of the susceptible class $\left(s_{0}^{k}=1-i_{0}^{k}\right)$. Hence the final size of the epidemic, given by (12), will be known. If the initial condition of the infected class is random (uncertain) the final size of the epidemic will also be random (uncertain).

\section{STATISTICAL PROPERTIES OF THE RANDOM SOLUTIONS}

The behaviour of the probability density function of the initial state of the infected class with known statistical properties will affect the behaviour of the probability density function of the random solution at any time. The determination of this family of distribution functions constitutes the solution that will be obtained numerically since the SIR model has no closed form solution. Determining the probability density function of the random solution enables us to determine a number of the statistical properties associated with the solution, such as the moments of the solution. The moments especially the mean and the variance are surely among the most important features associated with the random solution.

\section{The probability density function of the solution}

The infected density function supposes to be identical to a beta density function with particular parameters $\alpha$ and $\beta$ at the start of the epidemic $(t=0)$, and it begins as a right skewed because the initial mean proportion of infected is designed to be close to 0.20 . Since the total proportion of population size is equal to one, which is fixed for all time, and the initial state of recovered class is equal to zero, then the susceptible density function would be also identical to a beta density function with particular parameters $\beta$ and $\alpha$. In this situation, the susceptible density function begins as a left skewed and the initial mean proportion of susceptible would be nearby 0.80 . As time passes, the proportion of susceptible will decrease due to the unidirectional transition in the epidemic from susceptible to infected, which will increase and then decrease due to the unidirectional transition in the epidemic from infected to recovered class. Thus the density function of $s$ changes to a right skewed distribution and the density function of $i$ first changes to left skewed and then return to right skewed. The density function of $r$ starts after on unit of time with the right skewed and then changes to left skewed. Fig. 1 shows the changing of the probability density function of the infected class over time.

The parameters $\alpha$ and $\beta$ affect on the probability density function of the random solution of the model at any time, and depending on these two parameters, the density functions can take on different shapes. The probability density functions with the smallest values of $\alpha$ and $\beta$ have the largest variance, and with lower

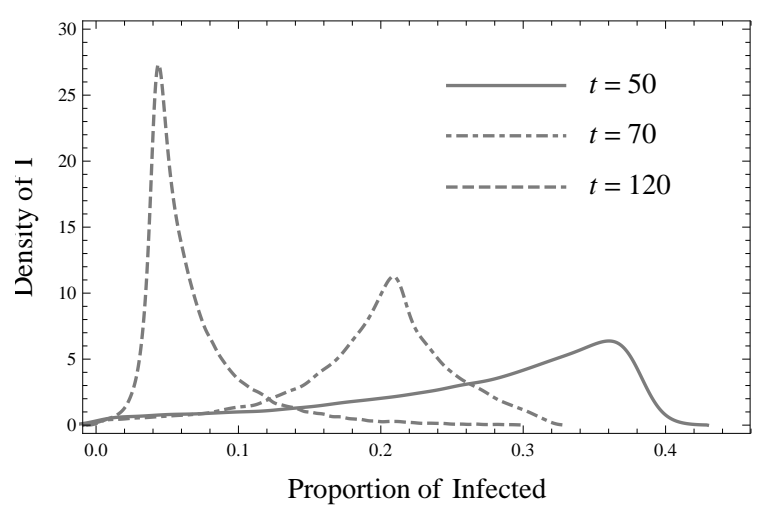

Fig. 1 Changing of density of the proportion of infectious over time with $\alpha=1$ and $\beta=4(\delta=1$ and $\gamma=1 / 3)$. 


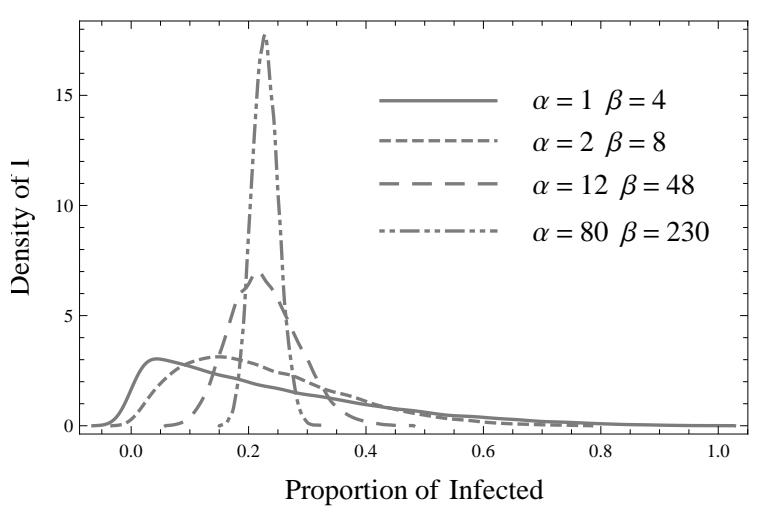

Fig. 2 The PDF of the proportion of infected with different values of $\alpha$ and $\beta$ at $t=3(\delta=1$ and $\gamma=1 / 3)$.

variance is the faster function shifts from left to right skewed or from right to left skewed. Fig. 2 illustrates the effect of the two parameters of a beta distribution on the probability density function of the proportion of infected class.

\section{The means of the proportion classes}

The mean of the random solution gives an average around which it realizes the solution. The means of the proportions of the three classes, susceptible, infected and recovered are also obtained numerically. Since the proportion of susceptible in the SIR epidemic model will decrease due to the unidirectional transition in the epidemic from susceptible to infected, which will increase in the beginning and then will decrease due to the unidirectional transition in the epidemic from infected to recovered, the mean proportion of susceptible will decrease over time as time passes, and the mean proportion of infected will increase and then decreased over time. The mean proportion of infected

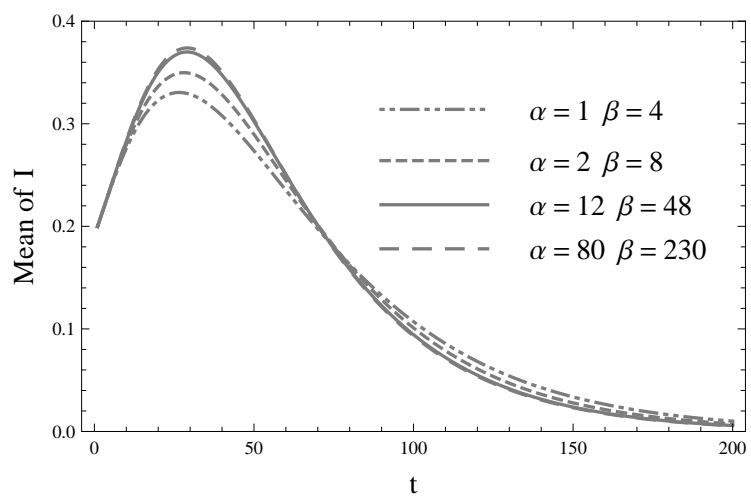

Fig. 3 Mean proportion of infected over time with different values of $\alpha$ and $\beta(\delta=1$ and $\gamma=1 / 3)$. will approach zero as time goes to infinity and the mean proportion of susceptible will approach $\left(\mu s_{\infty}>\right.$ 0 ) as time goes to infinity (after the epidemic). In addition, the mean proportion of recovered will increase over time and will also approach $\left(\mu r_{\infty}<1\right)$ after the epidemic. The behaviour of the mean proportion of infected over time for four different values of the parameters of $\alpha$ and $\beta$, where each of these parameters has the same initial mean proportion of infected $(0.2)$ is presented in Fig. 3. This figure shows us that the rate of decrease of the means proportion of the infected class to zero decreases as $\alpha$ and $\beta$ decrease. In addition, this rate decreases fastest for large $\alpha$ and $\beta$. The parameters $\alpha$ and $\beta$ have the same effect on the behaviour of the means proportions of susceptible and recovered over time and they reach their finite $\mu s_{\infty}$ and $\mu r_{\infty}$, respectively.

\section{The variances of the proportion classes}

The variance or the second moment of the random solution provides a measure of dispersion for possible values of the various realizations of the solution at any given point. The variances of the proportions of susceptible and infected are designed in this simulation to be similar, and equal to the variance of a beta distribution with the parameters $\alpha$ and $\beta$. The variance of a beta distribution is lower with the higher values of $\alpha$ and $\beta$. Thus the height or reduction of the initial variances of the proportions of susceptible and infected depends on the parameters $\alpha$ and $\beta$. As time passes, the curves of the variances of the proportions of susceptible and infected increase at the early values of $t$, and then decrease. Moreover, the curve of the variance of the proportion of recovered over time decreases at the early values of $t$, increases in the middle, and then again decreases. The curves of the variance of the proportion of infected reach to

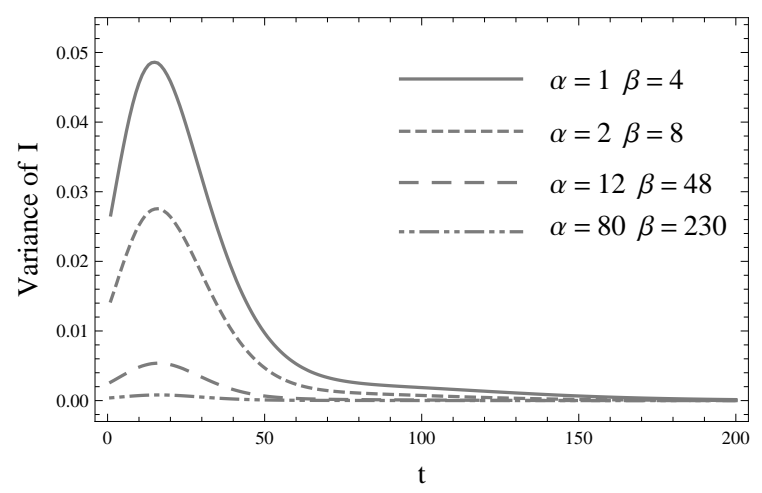

Fig. 4 Variance of the proportion of infected over time with different values of $\alpha$ and $\beta(\delta=1$ and $\gamma=1 / 3)$. 
zero at the later stage of the epidemic for all values of $\alpha$ and $\beta$, and the curves of the variances of the proportions of susceptible and recovered reach to their finite $\operatorname{Var}\left(s_{\infty}\right)$ and $\operatorname{Var}\left(r_{\infty}\right)$, respectively, at the end of the epidemic. Fig. 4 represents the behaviour of the variance of proportion of infected over time for different values of $\alpha$ and $\beta$. It shows us that, for the smaller values of $\alpha$ and $\beta$, the proportion have the larger initial variance. These smaller values maintain the larger variance over time. Furthermore, the rate of reaching the curves of the variance of the proportion of infected is faster for lower variance or by another words for the higher values of $\alpha$ and $\beta$. These results are applicable on the behaviour of the proportions of susceptible and recovered over time except at two things; first, the initial variance of recovered class assumed to be equal to zero. Secondly, the curves of the variances of these proportions do not reach zero at the end of the epidemic.

The interquartile range is the range of the middle $50 \%$ of values in a rank-ordered distribution. In other words, it gives the difference between the upper and lower quartiles for the distribution. Unlike the variance, the interquartile range does not take all of a variable's values into account when depicting the dispersion of those values. The curves of the interquartile ranges of the three proportion classes of the SIR epidemic model have the same types of behaviour over time as the curves of variances. the curves of the interquartile ranges of the proportions of susceptible and recovered reach to Range $\left(s_{\infty}\right)$ and Range $\left(r_{\infty}\right)$ at the end of the epidemic. The types of behaviour of the interquartile range of infectious class is presented in Fig. 5.

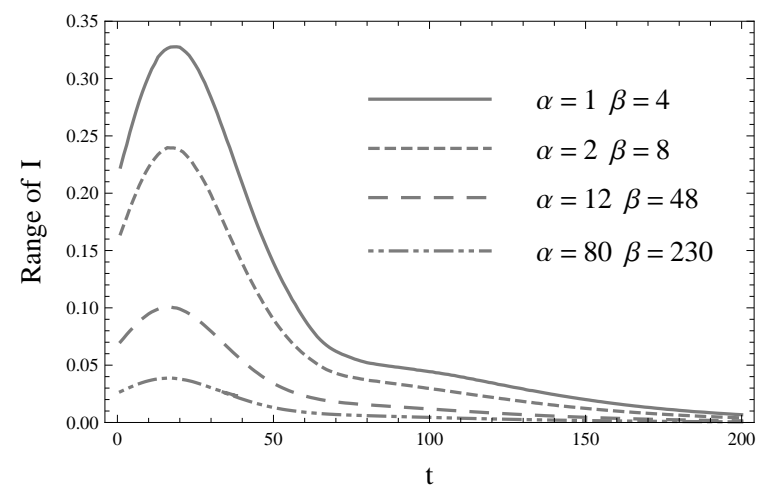

Fig. 5 Interquartile ranges of the proportion of infected over time with different values of $\alpha$ and $\beta(\delta=1$ and $\gamma=1 / 3)$.

\section{CONCLUSIONS}

Applications of differential equations model in epidemiological problems involve several levels of uncertainties. The SIR epidemic model of infectious diseases in populations is presented in this paper, and we have been concerned with the situation where there is uncertainty in the initial state of the proportion of infected individuals. A beta distribution was applied to be a random initial state of the proportion of infected individuals. Since this model is a nonlinear dynamical system and does not have a close solution, numerical simulations are used. Assuming a beta distribution as the initial state of the infected class affect on the final size of the epidemic that also becomes random (uncertain). Through the simulation, we considered how the parameters of a beta distribution affect the behaviour of the epidemic diseases. The results show us that the density probability functions of the three classes take many shapes during the epidemic, and the variety of $\alpha$ and $\beta$ affect on the fast or slow shifting of the densities functions from left to right skewed or right to left skewed. The means proportion of infected go to zero for all different values of $\alpha$ and $\beta$, and the rate of increase the mean for its maximum and decrease to zero is faster for small $\alpha$ and $\beta$. Moreover, the means proportions of susceptible and recovered go to their finite $\left(\mu s_{\infty}\right.$ and $\mu r_{\infty}$, respectively) for all different values of $\alpha$ and $\beta$. The variances and interquartile ranges of the proportion of the infected class also go to zero at the end of an epidemic for all different values of $\alpha$ and $\beta$. On another hand, the variances and interquartile ranges of the proportions of the susceptible and recovered classes reach their end values $\left(\operatorname{Var}\left(s_{\infty}\right)\right.$, Range $\left(s_{\infty}\right)$ and $\operatorname{Var}\left(r_{\infty}\right)$, Range $\left(r_{\infty}\right)$, respectively) which are greater than zero. The effect of the values of $\alpha$ and $\beta$ on the behaviour of the probability density functions of the random solution and the moments of these density functions during the epidemic is also studied. Choosing the values of $\alpha$ and $\beta$ affect the height or reduction of the variances of the random solution at any time, where smaller values of these parameters have larger variance which will affect the distribution of the solution at any time.

Acknowledgements: This study was supported by a grant from the School of Mathematical Sciences, Universiti Sains Malaysia.

\section{REFERENCES}

1. Kegan B, West RW (2005) Modeling the simple epidemic with deterministic differential equations and random initial conditions. Math Biosci 195, 179-93. 
2. Pollett P, Dooley A, Ross J (2010) Modelling population processes with random initial conditions. Math Biosci 223, 142-50.

3. Enszer J, Stadtherr M (2009) Verified solution method for population epidemiology models with uncertainty. Int J Appl Math Comput Sci 19, 501-12.

4. Hethcote HW (2000) The mathematics of infectious diseases. SIAM Rev 42, 599-653.

5. Jordan D, Smith P (2007) Nonlinear Ordinary Differential Equations: An Introduction for Scientists and Engineers, Oxford Univ Press.

6. De Vries G (2006) A Course in Mathematical Biology: Quantitative Modeling with Mathematical and Computational Methods, Society for Industrial Mathematics.

7. Chaharborj S, Bakar M, Fudziah I, Akma I, Malik A, Alli V (2010) Behavior stability in two SIR-style models for HIV. Int J Math Anal 4, 427-34.

8. Stilianakis NI, Perelson AS, Hayden FG (1998) Emergence of drug resistance during an influenza epidemic: Insights from a mathematical model. J Infect Dis 177, 863-73.

9. Ferguson N, Keeling M, Edmunds W, Gani R, Grenfell B, Anderson R, Leach S (2003) Planning for smallpox outbreaks. Nature 425, 681-5.

10. Small M, Tse C (2005) Clustering model for transmission of the SARS virus: application to epidemic control and risk assessment. Physica A 351, 499-511. 